\section{SOI: 1.1/TAS DOI: 10.15863/TAS International Scientific Journal Theoretical \& Applied Science}

p-ISSN: 2308-4944 (print) e-ISSN: 2409-0085 (online)

Year: $2016 \quad$ Issue: $6 \quad$ Volume: 38

Published: $30.06 .2016 \quad$ http://T-Science.org

SECTION 3. Nanotechnology. Physics.
Victor Goldade

Doctor of Science (Eng), Professor in Physics, Francisk Skorina Gomel State University,

Physical Department

Gomel, Belarus victor.goldade@gmail.com

Dmitry Lyubimov

$\mathrm{PhD}$ in mechanics Engineering center L \& Co, Ltd,

Surgut, Russia rostexx@rambler.ru

Kirill Dolgopolov

$\mathrm{PhD}$ in mechanics

Engineering center L \& Co, Ltd,

Surgut, Russia

lik.1ab061@gmail.com

\title{
QUANTUM MODEL OF CHARGE TRANSFER IN METAL-POLYMER ELECTRETS
}

Abstract: Possibility of building a quantum model of the electret state is analysed. A new approach applied in development of the metal-polymer electret model consists in presenting the process of metal transfer from an electrode to polymer as macroscopic quantum effect.

Key words: electret state, quantum-mechanical approach, exciton, wave function, Planck's constant.

Language: English

Citation: Goldade V, Lyubimov D, Dolgopolov K (2016) QUANTUM MODEL OF CHARGE TRANSFER IN METAL-POLYMER ELECTRETS. ISJ Theoretical \& Applied Science, 06 (38): 10-15.

Soi: http://s-o-i.org/1.1/TAS-06-38-3 Doi: crossef http://dx.doi.org/10.15863/TAS.2016.06.38.3

\section{INTRODUCTION}

Currently, along with the traditional electret formation technologies, technologically unsophisticated and non-energy-intensive methods have been developed for polymer materials polarization by their heat treatment in contact with metals. These methods allow to combine the product forming operations and electric polarization of polymer matrix in one technological process, thus increasing the performance characteristics of the products made of structural polymers [1].

Metal-polymer electrets (MPE) are a specific type of electrets formed in the process of polymer films contact with a pair of short-circuited electrodes made of dissimilar metals. When "electrode1polymer-electrode2" sandwich is heated to the polymer yield (or melting) temperature, thermally stimulated current (which density amounts to unitstens $\mathrm{pA} / \mathrm{cm}^{2}$ ) is induced in the external circuit connecting the electrodes. MPE are known for specific mechanism of their structure formation consisting in metal ions diffusion into the polymer matrix in viscous-flow state, their subsequent neutralization and coagulation of the formed atoms as clusters [2].
Phenomenological description of the electret effect is based on the results of fundamental researches conducted by the founders of the modern electret theory B. Gross, G.M. Sessler, J. van Turnhout, A.N. Gubkin, B.I. Sazhin, G.A. Luscheikin et al. [3-6]. The limited possibilities of the classical electrodynamics apparatus used for description of the charge transfer processes in polymer dielectrics is one of the main reasons for the lack of correct theoretical model of the electret state formation in polymers.

The relation between the dielectric conductivity and its microstructure predetermines availability of quantum mechanisms affecting the charge transfer processes and formation of electret states in the dielectric [7]. Multi-particle nature of such system complicates quantum-mechanical computations, thus, causing to remain at the level of qualitative description of the process of electret states occurrence.

A new approach applied by us in development of MPE model consists in presenting the process of metal transition from an electrode to polymer film as macroscopic quantum effect [8] which equation explicitly includes the Planck's constant. The article addresses the possibility of building a quantum model of MPE electret state which basic provisions 


\begin{tabular}{|c|c|c|c|c|c|c|}
\hline Impact Factor: & $\begin{array}{l}\text { ISRA (India) } \\
\text { ISI (Dubai, UAE } \\
\text { GIF (Australia) } \\
\text { JIF }\end{array}$ & $\begin{array}{l}=1.344 \\
=0.829 \\
=0.564 \\
=1.500\end{array}$ & $\begin{array}{l}\text { SIS (USA) } \\
\text { PИНЦ (Russia) } \\
\text { ESJI (KZ) } \\
\text { SJIF (Morocco) }\end{array}$ & $\begin{array}{l}=0.912 \\
=0.234 \\
=1.042 \\
=2.031\end{array}$ & $\begin{array}{l}\text { ICV (Poland) } \\
\text { PIF (India) } \\
\text { IBI (India) }\end{array}$ & $\begin{array}{l}=6.630 \\
=1.940 \\
=4.260\end{array}$ \\
\hline
\end{tabular}

can be applied to the processes of formation of practically all types of electrets.

\section{EXCITON WAVE FUNCTION}

In the first approximation, a metal surface can be represented as the ordered collection of positive ions that create their own negative image (polarized negative) in the polymer that comes into contact with it (Fig. 1.1, a). Polarized "reflection" of the metal ion in the polymer matrix can be considered as a quasiparticle with a negative charge that is equal in absolute value to the metal ion charge and effective mass equaling to the mass of such ion $[9,10]$.

b)

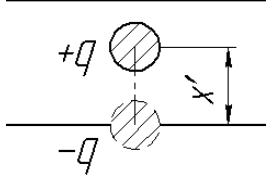

Figure 1 - Formation of charge states when a metal ion is transferred to the dielectric.

If the time period for metal ion transfer to the dielectric is less than the relaxation time, image $1, a$ is transformed as shown in Fig. 1, $b$. In other words, a negatively charged hole appears at the site of the metal ion and creates the already positively charged "reflection". From the standpoint of the quantum theory of solids, such system can be regarded as a bound dipole, or exciton, and the system transition from state $(1, a)$ into state $(1, b)$ can be interpreted as sequence of two turns of exciton by angle $\varphi=\frac{\pi}{2}$ [11].

Assuming that exciton of 1, $a$ type is described by wave function $\psi_{a}$, then wave function $\psi_{b}$ which describes the exciton after angular movement can be obtained from the unitary transformation as [11]:

$$
\psi_{b}=U^{-1} \psi_{a}=e^{-\frac{i \varphi}{\hbar}(\mathbf{n M})} \psi_{a},
$$

where $U^{-1}$ is the unitary operator, $\varphi$ is the exciton turn angle, $\hbar$ is the Planck's constant, $\mathbf{M}$ is the intrinsic angular momentum (spin), $\mathbf{n}$ is the normal to the surface. Absolute value of angular momentum M is related to Pauli spin matrix $\sigma$ as ${ }_{M=\frac{\text { oh }}{2}}^{2}$ [12].

Equation (1) can be re-written as follows:

$$
\psi_{b}=\left\{\cos \left[\frac{\varphi}{2}(n \sigma)\right]-i \sin \left[\frac{\varphi}{2}(n \sigma)\right]\right\} \psi_{a} .
$$

Since the substance particles are predominantly fermions, then $n \sigma=1$ [10] for them, so:

$$
\psi_{b}=\left\{\cos \frac{\varphi}{2}-i \sin \frac{\varphi}{2}\right\} \psi_{a} .
$$

The statement of the problem permits us to restrict ourselves to just the real part of the proportion (2):

$$
\operatorname{Re} \psi_{b}=\cos \frac{\varphi}{2} \psi_{a} .
$$

Then the first and second turns of exciton by 90 degrees shall be described by wave functions as:

$$
\begin{aligned}
& \psi_{b}^{\prime}=\cos \frac{\pi}{4} \psi_{a}=\frac{1}{\sqrt{2}} \psi_{a}, \\
& \psi_{b}=\cos \frac{\pi}{4} \psi_{b}^{\prime}=\frac{1}{2} \psi_{a} .
\end{aligned}
$$

Let us assume that wave functions $\psi_{a}$ and $\psi_{b}$ are "plane waves", i.e. $\quad \psi_{a}=a \exp \left(-\frac{i W_{a} t}{\mathrm{~h}}\right) \quad$ and $\psi_{b}=b \exp \left(-\frac{i W_{b} t}{\hbar}\right)$, where $W_{a}$ and $W_{b}$ are energies of the plane waves. If their amplitude vales are equal $(a=b)$, and taking into account (4) we obtain: $\quad 2 \exp \left(-\frac{i W_{b} t}{\hbar}\right)=\exp \left(-\frac{i W_{a} t}{\hbar}\right)$. Taking the logarithm of the reduced equation brings us to: $W_{a}=W_{b}+i \frac{\hbar}{t} \ln 2$. Since $\ln 2 \approx 0.7$, the resultant equation will be as follows:

$$
W_{a}-W_{b}=0,7 i \frac{\hbar}{t} .
$$

Equation (5) allows to consider the value $0,7 i \frac{\mathrm{h}}{t}$ as excitation $V$, and since the energy is a real value, it is possible to take instead of $0,7 i \frac{\mathrm{h}}{t}$ ) only the $\operatorname{Im} V$, i.e. $0,7 \frac{\mathrm{h}}{t}$. From the mathematical point of view, transition to only real component in the right-hand part of equation (5) is not quite correct, but in view of the laws of physics it might be considered as a part of the underexponential equation corresponding to the plane waves energy.

The energy of the exciton charges interaction is determined by the known formula [13]:

$$
W=-\alpha \frac{e^{2} n \Omega^{2}}{x^{4}}\left(\frac{\varepsilon-1}{\varepsilon+2}\right)^{2},
$$

where $\alpha$ and $\varepsilon$ are the dielectric susceptibility and dielectric permittivity of the medium respectively, $e$ is the electron charge, $n$ is the particles 


\begin{tabular}{|c|c|c|c|c|c|c|}
\hline Impact Factor: & $\begin{array}{l}\text { ISRA (India) } \\
\text { ISI (Dubai, UAE } \\
\text { GIF (Australia) } \\
\text { JIF }\end{array}$ & $\begin{array}{l}=1.344 \\
=0.829 \\
=0.564 \\
=1.500\end{array}$ & $\begin{array}{l}\text { SIS (USA) } \\
\text { PИНЦ (Russia) } \\
\text { ESJI (KZ) } \\
\text { SJIF (Morocco) }\end{array}$ & $\begin{array}{l}=0.912 \\
=0.234 \\
=1.042 \\
=2.031\end{array}$ & $\begin{array}{l}\text { ICV (Poland) } \\
\text { PIF (India) } \\
\text { IBI (India) }\end{array}$ & $\begin{array}{l}=6.630 \\
=1.940 \\
=4.260\end{array}$ \\
\hline
\end{tabular}

concentration, $\Omega$ is the volume taken by them, $x$ is the distance between charges.

In accordance with the basic provisions of the quantum theory of solids, particles concentration $n$, as well as the probability density, can be presented as the squared absolute value of the wave function $\psi^{2}$ [14]. Indeed, the wave function or the wave vector (a.k.a. vector of state), in its broadest sense, characterizes a certain information field which comprehensively determines the physical system. Probability interpretation of the wave function was proposed by M. Born and does not exhaust its complete physical meaning. In the Sommerfeld quantum theory of metals (see, e.g., [15]) the wave function describes the particles density as well (i.e. their quantity or concentration). Besides, this concept does not contradict the Born's interpretation, since if the state is more probable, then, naturally, there are more particles in it.

Then, we can write the following expression for interaction energies:

$$
\begin{aligned}
& W_{a}=-\alpha \frac{e^{2} \psi_{a}^{2} \Omega^{2}}{x^{4}}\left(\frac{\varepsilon-1}{\varepsilon+2}\right)^{2}, \\
& W_{b}=-\alpha \frac{e^{2} \psi_{b}^{2} \Omega^{2}}{x^{4}}\left(\frac{\varepsilon-1}{\varepsilon+2}\right)^{2} .
\end{aligned}
$$

By substituting the values of physical quantities from equations (4) and (7) into expression (5) and by taking simple transformations, we obtain:

$W_{a}-W_{b}=\frac{3}{4} W_{a}=\frac{3}{4} \frac{e^{2} \psi_{a}^{2} \Omega^{2}}{x^{4}}\left(\frac{\varepsilon-1}{\varepsilon+2}\right)^{2}=0,7 \frac{\hbar}{t}$

(according to (5)).

As it has been shown in [16], electrical conductivity of polymers in contact with metals is determined by disintegration energy $W_{d}$ of ionized molecules that characterizes the accumulation depth of the structural defects which may function as electron traps:

$$
\gamma=\gamma_{0} \exp \left(-\frac{W_{d}}{k T}\right)
$$

where $\gamma_{0}$ is the constant temperature-independent multiplier, $k$ is the Boltzmann constant, $T$ is temperature.

The value of time interval $t$ in formula (5) is close to the value of relaxation time $\tau=\varepsilon \gamma$, where $\gamma$ is the conductivity of the medium. Let's substitute time $t$ with relaxation time $\tau$ in equation (8), and the exponent from formula (9) with the Maclaurin series. Then:

$$
\begin{aligned}
& \psi_{a} \approx \frac{30}{x^{\prime}}\left(\frac{\varepsilon+2}{\varepsilon-1}\right) \sqrt{\frac{\gamma_{0}}{\varepsilon}}\left(1-\frac{W_{d}}{2 k T}\right), \\
& \psi_{b} \approx \frac{15}{x^{\prime}}\left(\frac{\varepsilon+2}{\varepsilon-1}\right) \sqrt{\frac{\gamma_{0}}{\varepsilon}}\left(1-\frac{W_{d}}{2 k T}\right) .
\end{aligned}
$$

where $x^{\prime}=\alpha x$.

The form of expressions for wave functions describing the exciton rotation (Fig. 1) corresponds to spherical waves which can be interpreted as occurrence of excitations of the internal electromagnetic fields in the exciton system associated with the relaxation processes of polarization charges redistribution. The basis for these processes is the principle of "energy expediency" which is implemented in a specific way on each occasion. In our case, it is realized as the potential equalization inside the dielectric material caused by the charge transfer described by the continuity equation:

$$
\frac{\partial n}{\partial t}+\operatorname{div} j=0
$$

where $j$ is the charge flow which can be expressed via wave functions [11]:

$$
j=\frac{i \hbar}{2 m}\left(\psi \frac{\partial \psi^{*}}{\partial x^{\prime}}-\psi^{*} \frac{\partial \psi}{\partial x^{\prime}}\right)
$$

where $m$ is the effective mass of the charged particles system, $\psi^{*}$ is the conjugate value of the wave function.

Description of such processes requires introduction of certain assumptions on time dependencies that are implicitly available in equations (10) for the exciton wave functions. Since all processes of the charge transfer and redistribution in the system under study are limited by relaxation time $\tau$, it is justifiable to introduce a corresponding chronal characteristic by means of Dirac $\delta$-function [14]:

$$
\begin{aligned}
& \psi=\frac{\psi_{0}}{x^{\prime}} e^{-i A \delta(t-\tau)}, \\
& \psi^{*}=\frac{\psi_{0}}{x^{\prime}} e^{i A \delta(t-\tau)} .
\end{aligned}
$$

where $A$ is the a dimensional constant.

Substitution of ratios (13) into equation (12) produces the following result:

$$
j=\frac{i \mathrm{~h}}{2 m}\left(-\frac{\psi_{0}}{x^{\prime}} \frac{\psi_{0}}{x^{\prime 2}}+\frac{\psi_{0}}{x^{\prime}} \frac{\psi_{0}}{x^{\prime 2}}\right)=0
$$

Then, in accordance with the continuity equation (11) it turns out that $\frac{\partial n}{\partial t}=0$, and, consequently, $n=$ const, meaning that the number of particles per unit volume of the dielectric phase space is constant which is supported by the balance between the particles inflow and outflow observed in the charge transfer process. 


\begin{tabular}{|c|c|c|c|c|c|c|}
\hline Impact Factor: & $\begin{array}{l}\text { ISRA (India) } \\
\text { ISI (Dubai, UAE } \\
\text { GIF (Australia) } \\
\text { JIF }\end{array}$ & $\begin{array}{l}=1.344 \\
=0.829 \\
=0.564 \\
=1.500\end{array}$ & $\begin{array}{l}\text { SIS (USA) } \\
\text { PИНЦ (Russia) } \\
\text { ESJI (KZ) } \\
\text { SJIF (Morocco) }\end{array}$ & $\begin{array}{l}=0.912 \\
=0.234 \\
=1.042 \\
=2.031\end{array}$ & $\begin{array}{l}\text { ICV (Poland) } \\
\text { PIF (India) } \\
\text { IBI (India) }\end{array}$ & $\begin{array}{l}=6.630 \\
=1.940 \\
=4.260\end{array}$ \\
\hline
\end{tabular}

From the aforementioned, it follows that if realquantitative changes inside the phase space do not exert any significant influence upon relaxationstructural behavior of the components of "dielectricmetal" physical system, the need remains to examine the chronal (temporal) relations between these processes.

\section{CHARgE TEXTURE}

Temporal, as well as any other coordinate transformations in quantum mechanics are defined by unitary operators $[11,14]$ :

$$
\begin{aligned}
& \psi_{i}(t)=U_{i}(t) \psi(0), \\
& U_{i}(t)=\exp \left(-\frac{i}{\hbar} H_{i} t\right),
\end{aligned}
$$

where $U(t)$ is the unitary time-shift operator, $H_{i}$ is the Hamiltonian of a system with its own set of energy values $W_{i}$.

In accordance with the foregoing, when time values match the relaxation time $\tau$, dynamic structures (excitations) associated with the flows of charged particles, as well as the flows themselves, disappear which means zeroing of their wave functions:

$$
\psi_{i}=\frac{\psi_{0}}{x^{\prime}} e^{-\frac{i}{h} W_{i}(t-\tau)}=0,
$$

or in accordance with equations (10):

$$
\frac{\psi_{0}}{x^{\prime}}=\frac{30}{x^{\prime}}\left(\frac{\varepsilon+2}{\varepsilon-1}\right) \sqrt{\frac{\gamma}{\varepsilon}}=0
$$

or in accordance with equation (13):

a)

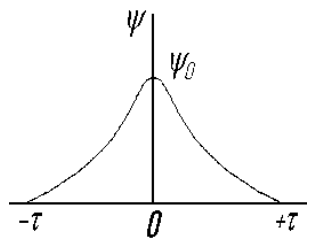

c)

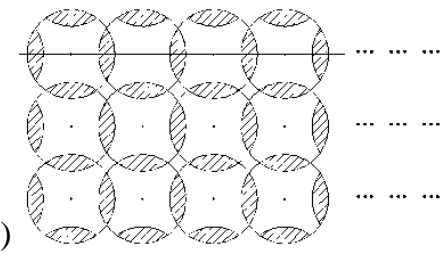

$\exp \left(-\frac{i}{\mathrm{~h}} W_{i}(t+\tau)\right)=\exp [-i A \delta(t-\tau)]_{t=\tau}=0$.

The fulfillment of condition (16) is implemented only in the case when $\varepsilon=-2$, which is impossible for all known natural substances. However, condition (17) is quite feasible, since the huge negative value of the exponent index of about $10^{45}$ (which is obtained when the finite value is divided by the Planck's constant) can be regarded as the "vanishing" of the given physical quantity to zero, and the relaxation processes become dependent only on the time parameter.

The obtained expressions for the wave function (10) and (13) describing the event of charge transfer from the metal to the dielectric have a form of spherical wave which amplitude attenuates in time (Fig. 2, a). Such wavefront may propagate for a distance of not more than $c^{\cdot} \tau$, where $c$ is the speed of light. Since there are many waves of that kind, then, as the result of merging of several wavefronts, areas $\Sigma$ are formed with higher values of the probability density (wave functions) (Fig. 2, $b$ ). Owing to spherical symmetry of the wave functions, areas $\Sigma$ produce the structures which form is close to crystalline one (Fig. 2,c) and which are turned by 45 degrees relative to the initial position of the exciton (Fig. 2, $d$ ). Such areas correspond to higher values of charge densities, since high $\psi^{2}$ values are indicative of high electron densities and, consequently, of possibility to form strong bonding orbitals. In relation to the internal volume of the solid body in question, this is equivalent to establishment of strong cohesive bonds and formation of any quasicrystalline, e.g. cluster, structure.

Figure 2 - Model of formation of the metal-polymer electret charge texture.

Noteworthy is a fact that "the turn angle" of pseudocrystalline structure formed during the charge transfer matches the turn angle of the wave vector which can serve as proof of correctness of the 
proposed physical model. Arrangement of the pole signs of the structure being formed (Fig. 2, d) provide additional "stability" to the latter which demonstrates that the given physical system is capable of forming the electret states.
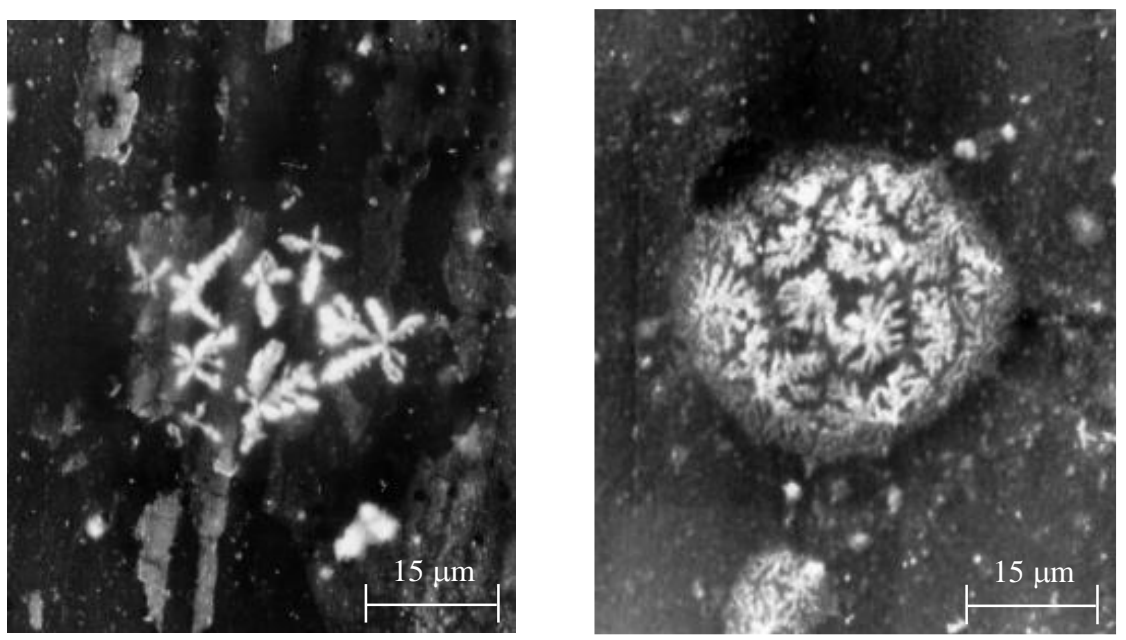

Figure 3 - Morphology of Ni particles in the polymer (polyvinyl butyral) matrix.

Our previously obtained experimental results on metal diffusion into the polymeric matrix during metal-polymer electret formation [17] can evidence as an indirect confirmation of the proposed model. It was shown by electron microscopy that colloid metal particles of dendritic and globular shape (of 2-5 $\mu \mathrm{m}$ ) are forming in the near-electrode layer of the polymeric matrix (Fig. 3). This enabled us to affirm that metal may be in the polymer matrix in the form of clusters forming a distinctive quasi-crystalline structure.

\section{CONCLUSION}

A new approach was applied in development of physical model of metal-polymer electret which consists in presenting the process of metal transition from electrodes to polymer phase as the macroscopic quantum effect which equation explicitly includes the Planck's constant. Metal ions transfer into dielectric is regarded as occurrence of a bound dipole, or exciton. The expressions for wave functions describing the exciton rotation are obtained which can be interpreted as occurrence of excitations of the internal electromagnetic fields in the exciton system associated with the relaxation processes of polarization charges redistribution in the polymer dielectric. Owing to spherical symmetry of the wave functions, areas with higher values of the probability density can be presented in space as structures resembling the crystalline form which are turned by 45 degrees relative to the initial position of the exciton.

\section{References:}

1. Kestelman V (2000) Electrets in Engineering: Fundamentals and applications / V. Kestelman L. Pinchuk, V. Goldade. - Boston, Dordrecht, London: Kluwer Academic Publ., 2000. - 281 p.

2. Belyi VA (1979) Electret state of polymers in metal-polymer-metal joint. / V.A. Belyi, V.A. Goldade, A.S. Neverov, L.S. Pinchuk // Proc. USSR AS, 1979, Vol. 245, No 1, pp. 132-134

3. Sessler GM (1987) Electrets. Topics in Applied Physics, Vol. 33, 2nd Ed. / G.M. Sessler, Ed. Berlin: Springer Verlag, 1987. - 385 p.

4. Gubkin AN (1978) Electrets / A.N. Gubkin. Moscow: Nauka, 1978. - 182 p.
5. Luscheikin GA (1984) Polymer electrets / G.A. Luscheikin. - Moscow: Khimiya, 1984. - 184 p.

6. Sazhin BI (1986) Electrical properties of polymers, $3^{\text {rd }}$ Ed. / B.I. Sazhin, Ed. Leningrad: Khimiya, 1986. - 224 p.

7. Kozakov AT (2009) Physical foundation of electron spectroscopy of solid charged surfaces / A.T. Kozakov. - Rostov-on-Don: Publ. of Russian South Federal University, 2009. - 406 p.

8. Pitaevski LP (1992) Macroscopic quantum effects / L.P. Pitaevski // In Physical 


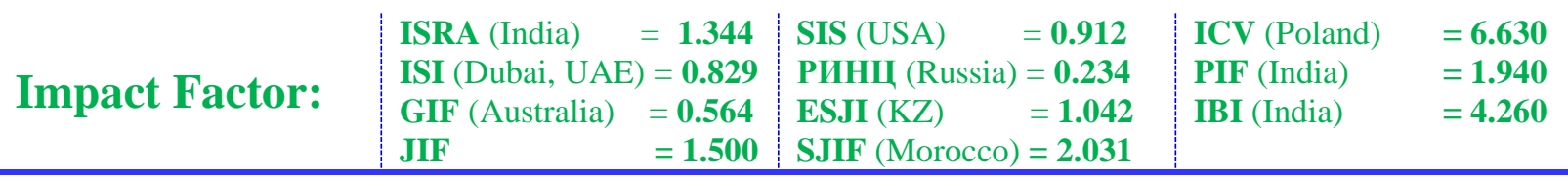

Encyclopedia. - Moscow: Russian Encyclopedia, 1992, Vol. 3, pp. 29-31.

9. Zangwill A (1988) Physics at surfaces / A. Zangwill. - Cambridge: University Press, 1988. $-472 \mathrm{p}$.

10. Lyubimov DN (2012) Quantum tribophysics / D.N. Lyubimov, L.S. Pinchuk, K.N. Dolgopolov. - Rostov-on-Don: Publ. of Russian South Federal University, 2012. - 292 p.

11. Medvedev BV (1977) Principles of theoretical physics / B.V. Medvedev. - Moscow: Nauka, 1977. - $496 \mathrm{p}$.

12. Pavlov VP (1992) Pauli matrixes / V.P. Pavlov // In Physical Encyclopedia. - Moscow: Russian Encyclopedia, 1992, Vol. 5, p. 550.

13. Tamm IE (1989) Fundamentals of electricity theory / I.E. Tamm. - Moscow: Nauka, 1989. $504 \mathrm{p}$.
14. Landau LD (1989) Theoretical physics, Vol. 3. Quantum mechanics (nonrelativistic theory) / L.D. Landau, E.M. Lifshits. - Moscow: Nauka, 1989. $-768 \mathrm{p}$.

15. Kittel Ch (2005) Introduction to solid state physics, $8^{\text {th }}$ Ed. / Ch. Kittel. - NJ: Wiley, 2005. $-703 \mathrm{p}$.

16. Goldade VA (1987) Electret plastics: Physics and material science / V.A. Goldade, L.S. Pinchuk. - Minsk: Nauka i tekhnika, 1987. $231 \mathrm{c}$.

17. Goldade VA (1999) Polarizing mechanism of colloidal metal particles formation in polymer electrets / V.A. Goldade, L.S. Pinchuk, E.A. Tsvetkova // In Proc. $10^{\text {th }}$ Int. Symp. on Electrets. - Delphi (Greece), 1999, p. 35-38. 